\title{
Measurements of the inclusive top quark pair production cross section in pp collisions at 7 and 8 TeV with CMS
}

\author{
Michael Maes* \\ Vrije Universiteit Brussel \\ On behalf of the CMS Collaboration \\ E-mail: michael.maes@cern.ch
}

\begin{abstract}
Precision measurements are presented of the top-quark pair inclusive production cross section in proton-proton collisions at the LHC at centre-of-mass energies of $7 \mathrm{TeV}$ and $8 \mathrm{TeV}$. The data are collected with the CMS experiment during the years 2011 and 2012. The analyses include all top quark pair final states with the exception of events with two tau-leptons in the final state. In most analyses b-jet identification is used to increase the purity of the selection. The backgrounds are determined using data-driven techniques. The results are combined with each other and compared with theory predictions.
\end{abstract}

The European Physical Society Conference on High Energy Physics -EPS-HEP2013

18-24 July 2013

Stockholm, Sweden

\footnotetext{
*Speaker.
} 


\section{Introduction}

The top quark, discovered in 1995 at the Tevatron Collider, plays a crucial role in the Standard Model (SM) since it is the heaviest known fundamental particle. As the top quark is expected to couple strongly to particles predicted in Beyond the Standard Model theories, the study of its properties is a key ingredient in the search for new phenomena. In 2011, the Large Hadron Collider delivered a dataset of $5 \mathrm{fb}^{-1}$ at a center-of-mass energy of 7TeV to the CMS experiment [1] followed in 2012 by a dataset of $20 \mathrm{fb}^{-1}$ at $8 \mathrm{TeV}$. The resulting large samples of top quark pairs provides a unique opportunity to measure the top quark pair production cross section with great precision. This is not only useful as a benchmark for QCD perturbative calculations, it is also expected to be sensitive to possible new physics phenomena.

The top quark decays predominantly in a $\mathrm{W}$ boson and a b quark. Given that the $\mathrm{W}$ boson decays either leptonically into a $l v_{l}$ pair either hadronically into a $q \bar{q}^{\prime}$ pair, this leaves three different decay modes for top quark pairs: semi-leptonic or $l+j e t s$ events, dilepton $t \bar{t}$ events and fully hadronic $t \bar{t}$ events. Measurements of the top quark pair cross section in the different channels will be presented in this proceeding and compared among each other and to the state-of-the-art theory predictions.

\section{Top quark pair cross section at $7 \mathrm{TeV}$}

The most precise measurement of the top quark pair production cross section is measured at $7 \mathrm{TeV}$ is performed in the dilepton channel with electrons and muons [2]. Using a dataset of 2.3 $f b^{-1}$, a cross section of $161.9 \pm 2.5$ (stat. $)_{-5.0}^{+5.1}($ syst. $) \pm 3.6$ (lum. $) p b$ is measured with a relative precision of ${ }_{-4.1}^{+4.2} \%$. Thanks to the presence of two leptons in the final state, dilepton $t \bar{t}$ events form a clear signal with a relatively low background. Events are selected by requiring 2 isolated leptons and at least two jets. To reduce the Z+jets background, $\mu^{+} \mu^{-} / e^{+} e^{-}$events are required to have a minimal Missing Transverse Energy $\left(\mathbb{E}_{T}\right)$ and have a dilepton mass outside of the $\mathrm{Z}$ boson mass window.

The $t \bar{t}$ signal is extracted from the distribution of the number of reconstructed jets and the number of $\mathrm{b}$ jets, as shown in Figure 1, using a Profile Likelihood Ratio fit using data-driven estimates for the DY+jets and non-prompt lepton backgrounds. In the fit, the main systematics are treated as nuisance parameters hence improving the overall precision on the result. The residual uncertainty is dominated by Jet Energy Scale (1.8\%), Lepton efficiencies (1.7\%) and the branching fraction of the $\mathrm{W}$ boson (1.7\%).

Additionally, the cross section is measured in dilepton events containing a $\tau$ lepton [7]. The dilepton event selection is altered to require only one isolated muon or electron in addition to a hadronically decaying $\tau$ lepton. Three jets are required and among them at least one has to be b-tagged [4]. The signal yield is extracted from a binned Maximum Likelihood fit on the top quark mass reconstructed with the KINb method [5], shown in Figure 1, using a data-driven technique to estimate the fake- $\tau$ background. For up to $2.2 \mathrm{fb}^{-1}$ of data, a $t \bar{t}$ cross section of $143.0 \pm$ 14.0 (stat.) \pm 22 (syst.) \pm 3.0 (lum.) pb with a relative precision of $18.4 \%$ is obtained where the uncertainty is dominated by the Background estimation (10.8\%), $\tau$ lepton identification (6.3\%) and the Jet Energy Scale (5.8\%). 

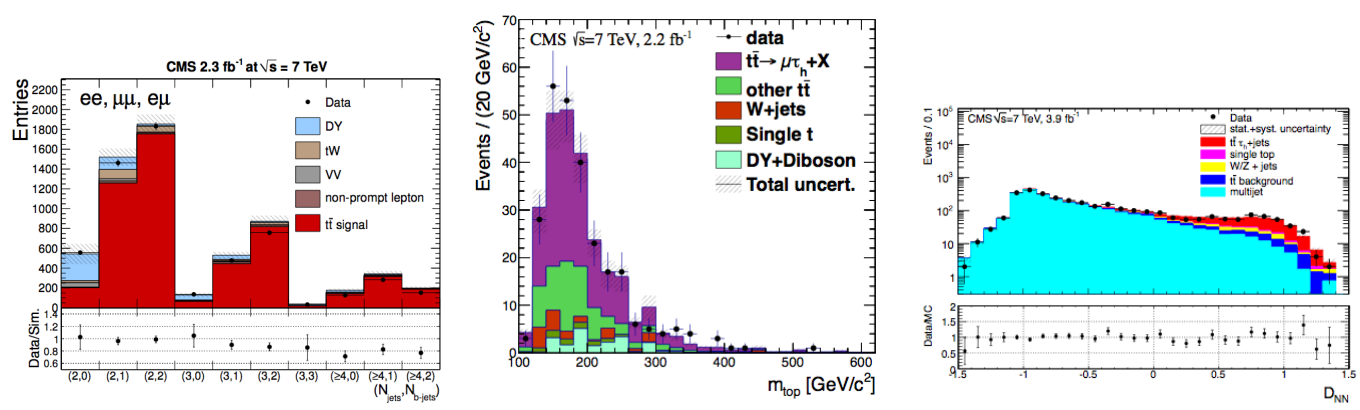

Figure 1: The number of reconstructed jets and the number of b-tags compared between data and simulation in the dilepton channel with muons and electrons (left). The top mass reconstructed in the $\mu \tau$ dilepton channel (middle). The neural network output separating $\tau+$ jets $t \bar{t}$ and background events (right).

Next, the $t \bar{t}$ cross section is measured in the $1+$ jets channel with $e / \mu+$ jets and $\tau+$ jets events respectively. In the $e / \mu+$ jets channel, the cross section is measured by using a Profile Likelihood Ratio fit on the mass of the Secondary Vertex in bins of the number of jets and the number of b-tagged jets [6]. Events are selected by requiring one isolated muon or electron in addition to four jets and a cut on $\mathbb{E}_{T}$. Since the number of reconstructed jets is sensitive to the Jet Energy scale and the number of $b$-tags to the b-tagging performance, both systematic uncertainties can again be treated as a nuisance parameters. A cross section of $158.1 \pm 2.1$ (stat.) \pm 10.2 (syst.) \pm 3.5 (lum.) pb is measured for a data sample of up to $2.3 \mathrm{fb}^{-1}$ providing a relative precision of $6.9 \%$. The main systematics in this measurement are Jet Energy Scale (2.4\%), b-tagging (2.1\%) and the W+jets $Q^{2}$-scale (1.6\%).

The cross section is also measured in the $1+$ jets channel using hadronically decaying tau leptons [7]. Although this channel is very difficult to use because of the vast multijet background, it is interesting because of the potential to be enriched by charged Higgs boson decays. Events are selected containing one hadronically decaying $\tau$ lepton together with four jets. One jet is required to be b-tagged. The background in this channel exists for over $90 \%$ out of multijet events which is estimated by a data-driven technique. A neural network based discriminator is put in place to separate the signal from the background. A $t \bar{t}$ cross section of $152.0 \pm 12.0$ (stat. $) \pm 32.0$ (syst.) \pm 3.0(lum.) $p b$ is obtained from a Maximum Likelihood fit on the neural network discriminator output displayed in Figure 1. The relative total uncertainty of $22.6 \%$ is dominated by the Jet Energy Scale (11\%), trigger (7\%), $\tau$ lepton identification $(9 \%)$ and $\not_{T}(7 \%)$ uncertainties.

Finally, the $t \bar{t}$ cross section is measured in the all hadronic channel [8] using $3.54 \mathrm{fb}^{-1}$ of data. Six or more jets are expected in each event. To reduce the abundant multijet background, at least two of those jets are required to be b-tagged using a high purity b-tagging operating point. The cross section is then measured using a maximum likelihood fit to the top quark mass while using a data-driven estimate for the multijet background. The top quark mass is reconstructed from the reconstructed jets after the proper matching between jets and partons is performed using a kinematic fit. The top quark pair production cross section in this channel is found to be $139.0 \pm$ 10.0 (stat. $) \pm 26.0$ (syst. $) \pm 3.0($ lum. $) p b$ with a precision of $20 \%$. 


\section{Top quark pair cross section at $8 \mathrm{TeV}$}

At a collision energy of $8 \mathrm{TeV}$, the top quark pair production cross section is up to now only measured in the $\mu \mu$, ee and $e \mu$ dilepton channels as well as the $e / \mu+$ jets semi-lepton channels. Just as with $7 \mathrm{TeV}$, the most precise result comes from the dilepton analysis [9] obtaining a total uncertainty of $6.7 \%$. Using a similar event selection strategy as for $7 \mathrm{TeV}$, the $t \bar{t}$ cross section is measured using a counting analysis using data-driven estimates for the DY+jets and non-prompt lepton backgrounds. For the combined dilepton channel, a cross section of $227.0 \pm 3.0$ (stat.) \pm 11.0 (syst.) \pm 10.0 (lum.) $p b$ is obtained for $2.4 \mathrm{fb}^{-1}$ of data where the Jet Energy Scale uncertainty $(3 \%)$ and the trigger and lepton efficiency uncertainties $(2 \%)$ are dominating the total uncertainty.

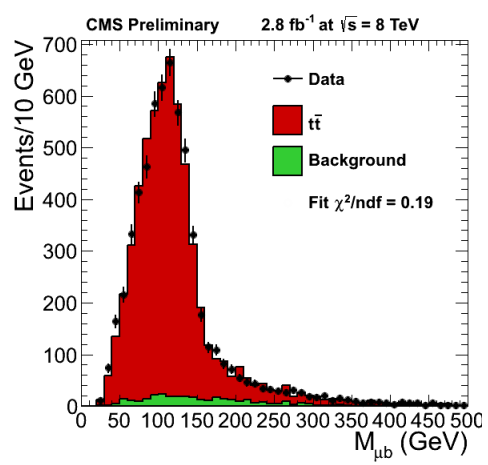

Figure 2: The jet-lepton mass distribution in the $\mu+$ jets channel.

In the $e / \mu+$ jets channel, the $t \bar{t}$ cross section is measured using a binned Maximum Likelihood fit on the invariant mass of the $\mathrm{b}$ jet candidate and the lepton in the $t \rightarrow l v_{l} b$ decay $\left(M_{l b}\right)$ [10] for which the distribution is shown in Figure 2 for the $\mu+$ jets channel. Events are selected by requiring exactly one isolated muon or electron in addition to at least four jets. The $b$ jet candidate is chosen among the four leading selected jets according to a $\chi^{2}$ jet sorting technique using the top quark and $\mathrm{W}$ boson masses. To suppress the background even further, the $\mathrm{b}$ jet candidate is required to be b-tagged. Finally, the b-tagging efficiency is measured in-situ with the cross section. This yields a measured value of $228.4 \pm 9.0$ (stat. $)_{-26.0}^{+29.0}($ syst.$) \pm 10.0$ (lum. $) p b$ with a relative uncertainty of $\sim 13.4 \%$ composed mainly of the Jet Energy Scale (5\%), b-tagging (8\%) and the $t \bar{t}$ signal modelling $(2-4 \%)$.

\section{Summary}

In Figure 3, the measured top quark pair production cross section is shown for all different decay modes at $7 \mathrm{TeV}$ (left) and $8 \mathrm{TeV}$ (right). At both centre-of-mass energies, the results are found to be consistent among the different channels. Moreover, the results are compared to the latest NNLO+NNLL theory predictions [11] (pink band) showing good agreement. At $8 \mathrm{TeV}$, the results are combined with the BLUE method albeit that the combination is dominated by the dilepton measurement because of its superior precision. As a consequence, the combination is not performed at $7 \mathrm{TeV}$ since here the dilepton result would also dominate. 

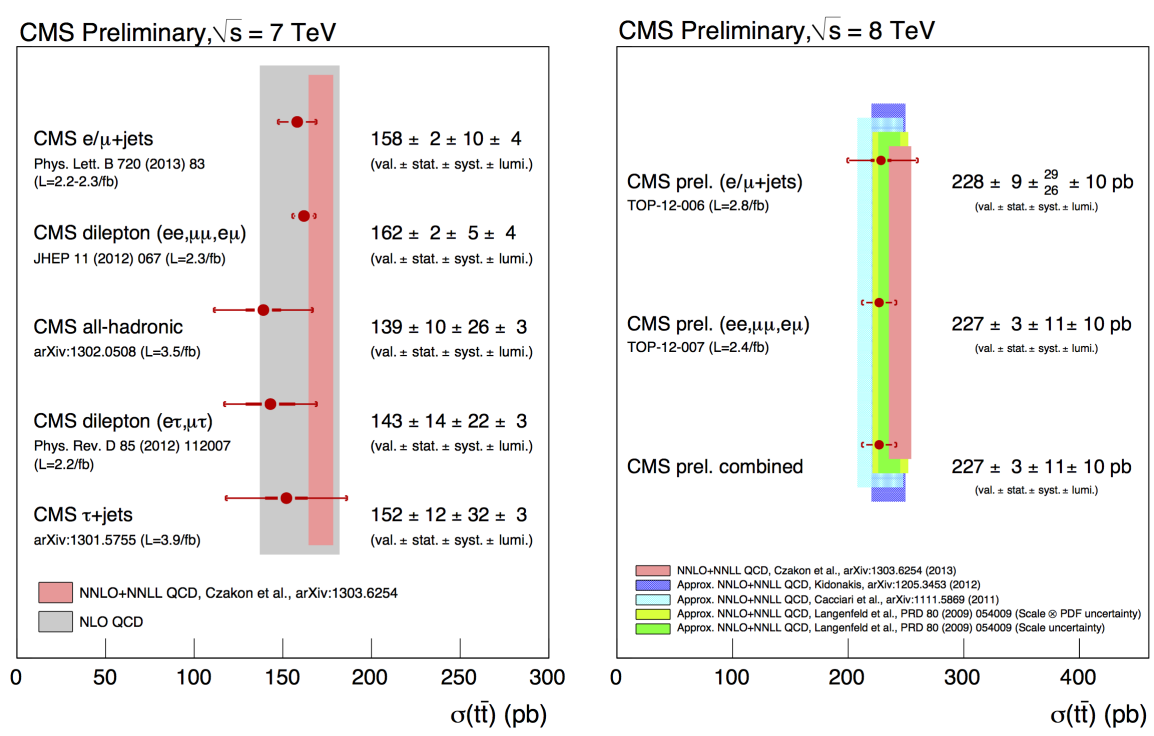

Figure 3: Measured top quark pair production cross section for all different decay modes at $7 \mathrm{TeV}$ (left) and $8 \mathrm{TeV}$ (right).

\section{References}

[1] CMS Collaboration, "The CMS experiment at the CERN LHC”, JINST 03 (2008) S08004.

[2] CMS Collaboration, "Measurement of the tt production cross section in the dilepton channel in pp collisions at $\sqrt{s}=7 \mathrm{TeV} "$, JHEP 11 (2012) 067. arXiv: 1208.2671.

[3] CMS Collaboration, "Measurement of the top quark pair production cross section in pp collisions at $\sqrt{s}=7 \mathrm{TeV}$ in dilepton final states containing a tau", Phys. Rev. D 85 (2012) 112007. arXiv: 1203.6810 .

[4] CMS Collaboration, “Identification of b-quark jets with the CMS experiment”, JINST 08 (2013) P04013. arXiv:1211.4462.

[5] CMS Collaboration, "Measurement of the $t \bar{t}$ production and the top-quark mass in the di-lepton channel in pp collisions at $\sqrt{s}=7$ TeV", JHEP 07 (2011) 049. arXiv: 1105.5661.

[6] CMS Collaboration, "Measurement of the top quark pair production cross section in pp collisions at $\sqrt{s}=7 \mathrm{TeV}$ with lepton + jets final states", Phys. Lett. B 720 (2013) 83. arXiv:1212.6682.

[7] CMS Collaboration, "Measurement of the top-antitop production cross section in the tau+jets channel in pp collisions at $\sqrt{s}=7 \mathrm{TeV}$ ', Eur. Phys. J. C 73 (2013) 2386. arXiv: 1301.5755.

[8] CMS Collaboration, "Measurement of the top-antitop production cross section in the all-jet final state in pp collisions at $\sqrt{s}=7 \mathrm{TeV}$, JHEP 05 (2013) 065. arXiv: 1302 . 0508.

[9] CMS Collaboration, “Top pair cross section in dileptons', CMS Physics Analysis Summary CMS-PAS-TOP-12-007 (2012).

[10] CMS Collaboration, “Top pair cross section in e/mu+jets at $8 \mathrm{TeV}$ ', CMS Physics Analysis Summary CMS-PAS-TOP-12-006 (2012).

[11] Czakon, Fiedler, Mitov, “ The total top quark pair production cross-section at hadron colliders through $\mathscr{O}\left(\alpha_{s}^{4}\right)$ ", Phys. Rev. Lett. 110 (2013) 252004. arXiv: 1303.6254. 